\title{
Modeling for Copper Ablation by Ultrashort Laser Bursts-Train
}

\author{
Ahmed Abdelmalek ${ }^{1}$, Zeyneb Bedrane ${ }^{1}$, El-Hachemi Amara ${ }^{2}$ \\ 1. Physics Department, Theoretical Physics Laboratory, Tlemcen University, Tlemcen 13000, Algeria \\ 2. Centre de Développement des Technologies Avancées, CDTA, PO. Box 17 Baba-Hassen, Algiers 16303, \\ Algeria \\ E-mail: ahmed.abdelmalek@univ-tlemcen.dz
}

Received: 24 April 2019; Accepted: 12 May 2019; Available online: 10 June 2019

\begin{abstract}
Ablation of copper using multipulse femtosecond laser irradiation with an 800 nm wavelength and 900fs pulse duration is investigated theoretically using a dynamic tow temperature model. Our results show that the irradiation of a metal film by burst femtosecond laser with a separation time between pulses less than the thermal relaxation time can dramatically enhance the irradiated focal volume without a significant dissipation of the energy inside the material. We demonstrate the advantage of burst irradiation at low fluence where the cooper can be ablated below single ablation threshold. We also suggest that at high fluence, irradiation with a burst-train may give rise to a cleaner ablation than with a pulse-train.
\end{abstract}

Keywords: Ultrashort laser; Burst; Two-temperature model; Fast thermal accumulation effect.

\section{Introduction}

The rapid development of pulsed lasers in recent years opens up broad areas of application in industry, materials science and medicine [1]. The laser-matter interaction involves many physical mechanisms, and its understanding requires taking into account, on the one hand, the parameters of the laser beam (energy, pulse duration, wavelength, etc.) and on the other hand, the physical properties of the material constituting the target.

It has been shown that the quality of ablation produced by a femtosecond laser (fs) is better than that due to a laser operating in nanosecond (ns) regime, or other relatively longer pulse in the domain of the picosecond (ps) [25]. Considerable improvement in micro-machining accuracy is possible with ultra-short lasers.

The physical mechanisms of femtosecond laser ablation are still under study. It has been observed that there are two different ablation phases during the transition from low fluence to higher fluence [5], i.e. gentle ablation and stronger ablation [6]. We seem that the physical mechanism changes when the ablation phases change, where at low fluence, electron-ion collision is the most dominant as proposed in our previous work [7], implying a gentle ablation, but at high fluence, electron-phonon collision is the most dominant [8], implying a strong ablation.

In fact, to produce ablation with great precision, it is necessary to apply the fluence as low as possible above the threshold to minimize damage to the material [4]. Then the problem is that at high fluence, drilling by femtosecond laser gives irregular craters by the formation of a corona on the surface contrary to the case of low fluence where the deposit is well minimized [9].

By taking the phase explosion as the dominant mechanism for ablation of a metal target irradiated by femtosecond laser pulses, the molten material can be overheated without boiling, because the time of relaxation is too short, so that a heterogeneous nucleation is produced. Therefore, when the temperature of the overheated liquids approaches the spinode (limit of heat of the metastable phase [10]), i.e. when the temperature is close to the critical temperature of the thermodynamic equilibrium $\mathrm{T}_{\mathrm{C}}$, the liquid tension tends rapidly to zero. As a result, homogenous nucleation occurs at an extremely high rate. The superheated liquid is expanded with the thermal explosion process and the material is a mixture of liquid and vapor droplets [10-12].

Ren et al. [13] and Huang et al. [14] simulated the ablation of a metals target using a burst of laser pulses with a separation time between pulses of 50 ps and 280 ps respectively. They found that at the same fluence, with this type of ablation, irradiation could dramatically increase the lattice temperature and the ablation rate compared to a single pulse.

Bursts with an extremely high repetition rate $(\mathrm{GHz})$ were proposed by Kerse et al. [15] for an efficient removal made possible.

At present work, we propose a fast thermal accumulation effect (FTAE) induced by bursts of femtosecond pulses with a repetition rate in the order of $\mathrm{GHz}$, ie. the separation time between the pulses must be shorter than the thermal relaxation time, which can considerably increase the lattice temperature in the irradiated focal volume, 
thus reducing the dissipation of energy inside material. As a result, there will be overheating of the liquid induced by the first pulse, which implies that the evaporation rate can be increased during the same phase explosion. As a result, a very high efficiency of drilling can be achieved at high fluence by this mode of irradiation.

\section{Two temperature model}

In the case of the interaction of a metal with an ultrashort pulse laser, because of the inverse Bremsstrahlung, the energy of the laser beam will be absorbed by the free electrons of the material, then a thermalization of electrons and energy transfer to the lattice via electron-phonon collision [16]. The energy transfer from the laser beam to the metal target such as copper can be described using a two-temperature model 1D-TTM, which considers the metal as being formed of two subsystems: a subsystem lattice characterized by its temperature $\mathrm{T}_{\mathrm{l}}$, and a subsystem electronic characterized by its temperature $\mathrm{T}_{\mathrm{e}}$.

In our simulation we will take copper as a target of thickness $d=3 \mu \mathrm{m}$ and at initial temperature $\mathrm{T}_{0}=300 \mathrm{~K}$ irradiated by ultrashort laser pulses of fluence $\mathrm{F}$ on the front surface $(\mathrm{z}=0)$.

The laser pulse is Gaussian in time with a Full Width at Half Maximum (FWHM) tp of 900 fs with a central wavelength of $800 \mathrm{~nm}$. The evolution of electron-lattice temperature can be described by the TTM [17,18]:

$$
\begin{aligned}
& C_{e}\left(T_{e}\right) \frac{\partial T_{e}}{\partial t}=\frac{\partial}{\partial z}\left(k_{e} \frac{\partial T_{e}}{\partial z}\right)-G\left(T_{e}-T_{l}\right)+S(z, t) \\
& C_{l} \frac{\partial T_{l}}{\partial t}=G\left(T_{e}-T_{l}\right)
\end{aligned}
$$

$\mathrm{C}$ is the heat capacity, $\mathrm{k}$ is thermal conductivity, where e and $\mathrm{l}$ denote electron and lattice, respectively. $\mathrm{G}$ is the electron-phonon coupling factor. $\mathrm{z}$ is the direction perpendicular to the target surface and $\mathrm{t}$ is the time.

The heat density $\mathrm{S}$ can be expressed for a multipulse as [8]:

$$
S(z, t)=\sum_{i=1}^{N} 0.94 \frac{1-R}{t_{p}\left(\delta+\delta_{b}\right)\left(1-e^{-\frac{d}{\delta+\delta_{b}}}\right)} F \cdot \exp \left[\frac{-z}{\left(\delta+\delta_{b}\right)}-2.77\left(\frac{t-(i-1) t_{s e p}}{t_{p}}\right)^{2}\right]
$$

where $N$ is the number of pulses per burst, $t_{\text {sep }}$ is the separation time between pulses, $\delta=1 / \alpha$ is the absorption depth, $\alpha$ is the absorption coefficient and $R$ the reflectivity. $\delta_{b}$ is the ballistic range. The ballistic range value has an important impact in the simulations since it provides the ballistic transport of energy by the hot electrons [17]. More details of this model and the copper parameters can be found in our previous work [8].

This dynamic TTM was simulated using MATLAB software by the finite differential method.

\section{Results and discussion}

\subsection{Calculation of optical parameters}

Figure 1 shows the reflectivity $\mathrm{R}$ and the absorption coefficient $\alpha$ calculated in function of temperature for a wavelength of $800 \mathrm{~nm}$. This figure indicates that $\mathrm{R}$ and $\alpha$ decrease considerably with the temperature. The decrease in $\mathrm{R}$ implies that the target absorbs more laser energy, whereas the decrease of $\alpha$ modifies the distribution of the laser's thermal density $\mathrm{S}$ within the target. It is obvious that these properties influence the amount of laser energy during irradiation, and then affect the thermal response of the material. This is why the optical dynamics must be taken into account during the simulation. Several works $[11,17,19]$ have considered $\mathrm{R}$ and $\alpha$ as constant, which can be valid only at low temperature.

\subsection{Reduction ablation threshold}

Figure 2 shows the evolution of sub-systems materials under the irradiation of train of pulses at $0.2 \mathrm{~J} / \mathrm{cm}^{2}$ with a repetition rate of $33 \mathrm{GHz}$, where $0.2 \mathrm{~J} / \mathrm{cm}^{2}$ is below single ablation threshold [18].

It can be seen in figure 2 that the thermal equilibrium between the electrons and the lattice is established after a few picoseconds, i.e. the lattice remains totally cold during the irradiation. We deduce then that the femtosecond laser-material interaction is a non-thermal interaction and that is why the femtosecond laser can allow more efficient drilling of materials.

We see also that the increase in the pulses number increases the temperature of the lattice but does not reach the thermal explosion condition, where the ablation of metals requires a lattice temperature close to $0.9 \mathrm{~T}_{\mathrm{C}}$ [17]. In copper the critical temperature is $\mathrm{T}_{\mathrm{C}}=7696 \mathrm{~K}[18]$. 
Therefore, to ablate the copper film we must exceed the critical temperature, for this reason, we proposed to irradiate the target by train of bursts instead of train of pulses where each burst contains a certain number of pulses.

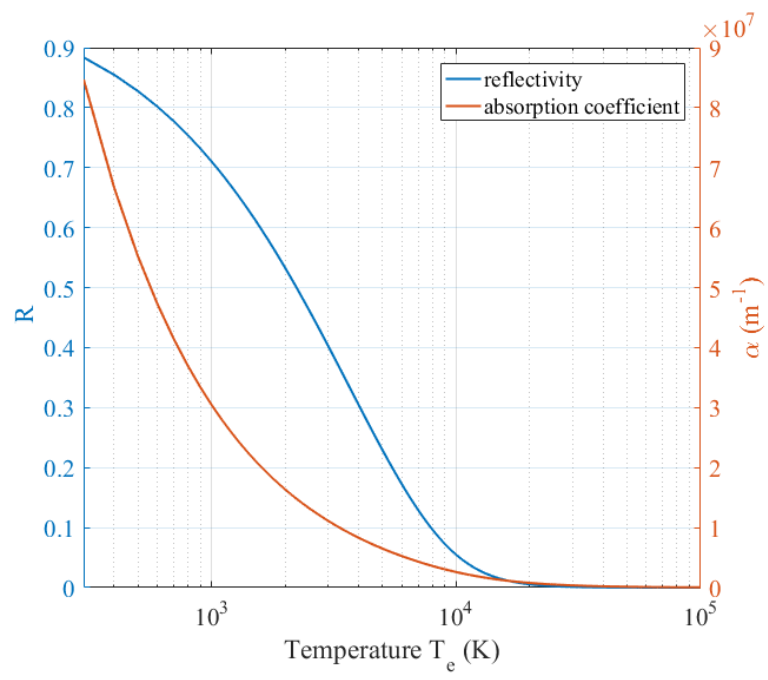

Fig. 1. Reflectivity and absorption coefficient versus electron temperature at $800 \mathrm{~nm}$ wavelength. Note that the $\mathrm{x}-$ axis starts at $300 \mathrm{~K}$.

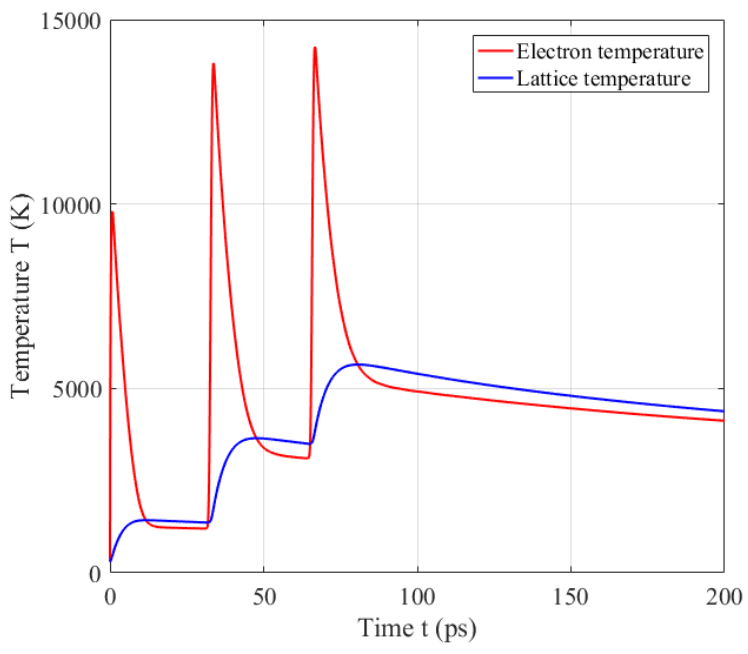

Fig. 2. Evolution of electron and lattice temperature as a function of time during the irradiation by train of pulse with a repetition rate of $33 \mathrm{GHz}, F=0.2 \mathrm{~J} / \mathrm{cm}^{2}$ and $800 \mathrm{~nm}$ of wavelength.

Figure 3 shows the evolution of sub-systems materials under the irradiation of train of bursts at $0.2 \mathrm{~J} / \mathrm{cm}^{2}$ with a repetition rate of $33 \mathrm{GHz}-200 \mathrm{GHz}$. Where $200 \mathrm{GHz}$ is the repetition rate of pulses for each burst and it correspond to $t_{\text {sep }}=5$ ps of separation time between pulses.

It is clear that lattice temperature is dramatically enhancement with train of bursts where the temperature exceeds the critical temperature at the end of irradiation. Therefore, the copper can be ablated at low fluence. This enhancement of temperature due to fast thermal accumulation effect (FTAE) during the irradiation because the separation time between pulses used is less than vibrational time scale which on order of picosecond. As conclusion, we deduce that the ablation threshold can be significantly reduced when irradiated the metals by train of bursts.

\subsection{Improved ablation at high fluence}

\subsubsection{Ablation by train of pulses}

As mentioned in the introduction, the ablation of metals by femtosecond laser pulse at high fluence called a strong ablation where a corona can be formed around craters, see Ref.[9]. Because the ablation at high fluence is mainly due to thermal explosion, then the material to be ejected in general as liquid shape, which decreases the surface quality of laser-induced microstructure. 
Figure 4(a) shows the evolution of sub-systems materials under the irradiation of train of pulse at $6 \mathrm{~J} / \mathrm{cm}^{2}$ with a repetition rate of $20 \mathrm{GHz}$ corresponding to 50 ps between pulses. We observe that the lattice temperature increases after each pulse due to the accumulation effect, and because of the time separation between pulses is much higher than the electron-phonon relaxation time; the matter will be ejected via phase explosion after each thermal relaxation. Therefore, in figure 4(a) there are three thermal explosions. The figure 4(b) show the lattice temperature versus time and position where we observed that after each pulse the material heated more and more, therefore, the superheated liquid exceeds the critical temperature and the phase explosion appeared. We notice that the ablation depth is below $1 \mu \mathrm{m}$.

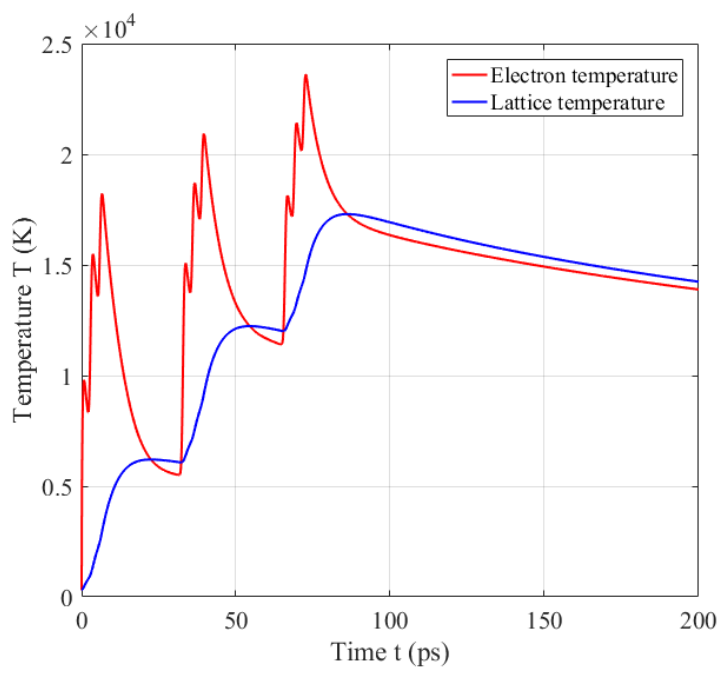

Fig. 3. Evolution of electron and lattice temperature as a function of time during the irradiation by train of burst with a repetition rate of $33 \mathrm{GHz}-200 \mathrm{GHz}, \mathrm{F}=0.2 \mathrm{~J} / \mathrm{cm}^{2}$ and $800 \mathrm{~nm}$ wavelength.

(a)

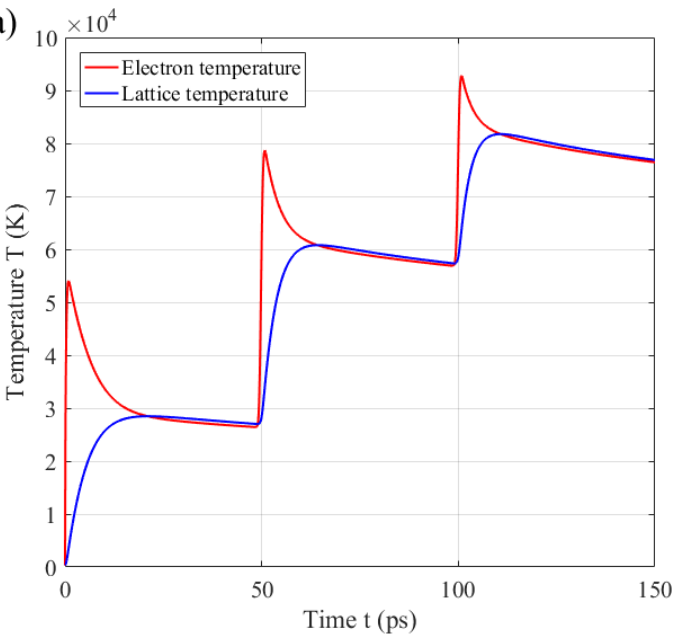

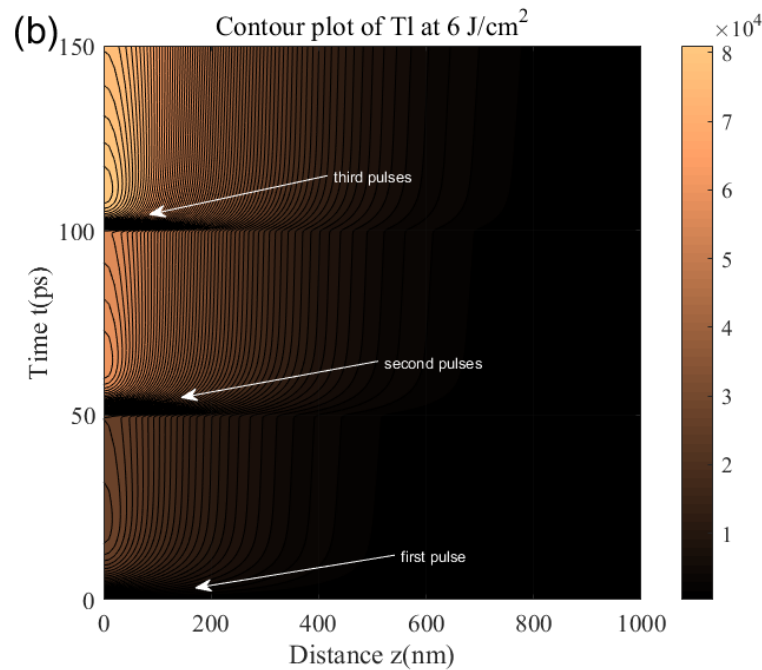

Fig. 4. (a) Evolution of electron and lattice temperature as a function of time; (b) Lattice temperature (Tl) versus time and position into a copper sample during the irradiation by train of pulse with a repetition rate of $20 \mathrm{GHz}, \mathrm{F}=6 \mathrm{~J} / \mathrm{cm}^{2}$ and $800 \mathrm{~nm}$ of wavelength.

\subsubsection{Ablation by train of burst}

Figure 5(a) shows the temporal evolution of sub-systems materials under the irradiation of train of bursts at 6 $\mathrm{J} / \mathrm{cm}^{2}$ with a repetition rate of $20 \mathrm{GHz}-200 \mathrm{GHz}$ corresponding to 50 ps between bursts and 5 ps between pulses respectively.

We observed that the lattice heated by train of bursts (figure 5(a)) reach a temperature values much higher compared to the case of irradiation by train of pulses (figure 4(a)). During the irradiation by femtosecond laser bursts, the lattice temperature enhancement under fast thermal accumulation effect (FTAE) because the separation time between pulses is less than thermal relaxation time. Therefore, we suggest that this mode of irradiation can enhance the temperature of the same focal volume irradiated by the first pulse. 
The figure 5(b) show the lattice temperature versus time and position where the lattice is more heated in comparing with train of pulses irradiation (see figure 4(b)). Although there is a great improvement in the heating of the irradiated focal volume, the ablated depth is almost the same, where it remains always below $1 \mu \mathrm{m}$.

Jia et al. [20] show that at high laser fluence, most of the laser energy absorbed consumed by material ejection and only $1 \%$ remains in the sample after ablation. In addition to this, we suggest basing on our current results that the ablation of copper at high fluence with train of burst mode enhance the superheated liquid at the same focal volume which can improve the vapour rate during the phase explosion in comparison with the liquid rate; therefore, a more clear drilling can be obtained.

(a)

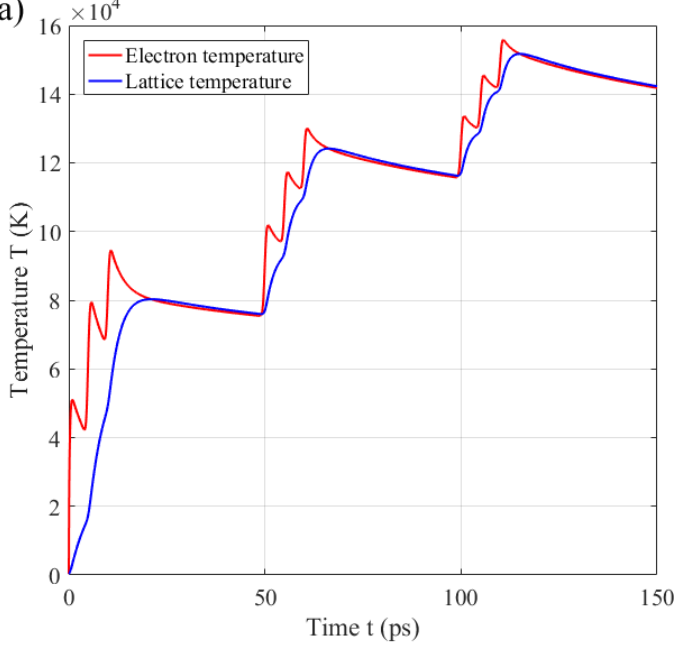

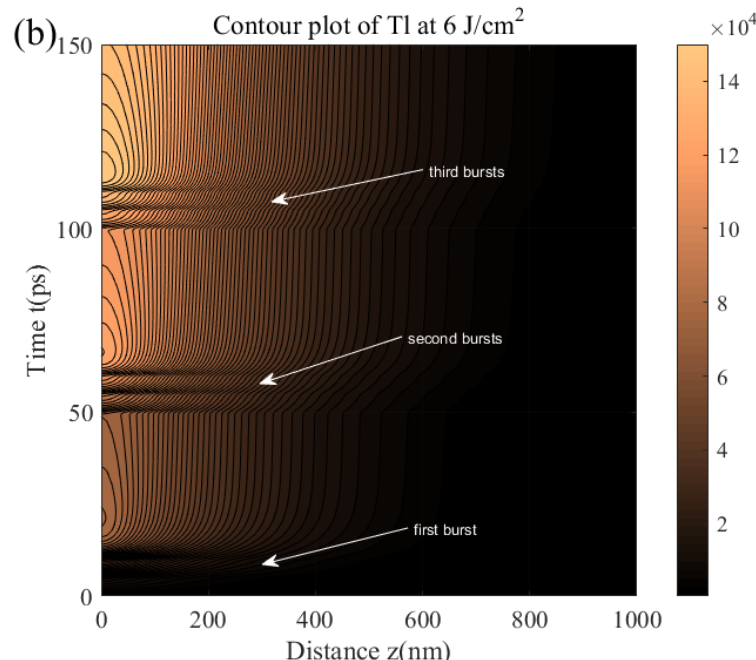

Fig. 5. (a) Evolution of electron and lattice temperature as a function of time; (b) Lattice temperature ( $\left.\mathrm{T}_{1}\right)$ versus time and position into a copper sample during the irradiation by train of burst with a repetition rate of $20 \mathrm{GHz}-$ $200 \mathrm{GHz}, \mathrm{F}=6 \mathrm{~J} / \mathrm{cm}^{2}$ and $800 \mathrm{~nm}$ of wavelength.

\section{Conclusion}

In this work, we have tried to show the advantage of the irradiation of metals by femtosecond laser burst-train where we have presented numerically a comparison in thermal response of copper during the irradiation between two mode of interaction; train of pulse and train of burst femtosecond laser. The two-temperature model adapted to the case of multipulses has been simulated by the finite difference method give a best visualisation of lasercooper interaction. The results obtained shows that the ablation threshold can be reduced by using a train of burst. It is clearly observed also that the temperature of the lattice increases considerably by burst-train irradiation compared to the case of the pulses-train irradiation, where we suggested that the quality of ablation could be improved by this mode of irradiation.

\section{References}

[1] Osellame R, Cerullo G, Ramponi R, editors. Femtosecond laser micromachining: photonic and microfluidic devices in transparent materials. Springer Science \& Business Media; 2012.

[2] Chichkov BN, Momma C, Nolte S, Von Alvensleben F, Tünnermann A. Femtosecond, picosecond and nanosecond laser ablation of solids. Applied Physics A. 1996;63(2):109-115.

[3] Semerok A, Salle B, Wagner JF, Petite G. Femtosecond, picosecond, and nanosecond laser microablation: Laser plasma and crater investigation. Laser and Particle Beams. 2002;20(1):67-72.

[4] Momma C, Nolte S, Chichkov BN, Alvensleben FV, Tünnermann A. Precise laser ablation with ultrashort pulses. Applied Surface Science.1997;109:15-19.

[5] Nolte S, Momma C, Jacobs H, Tünnermann A, Chichkov BN, Wellegehausen B, Welling H. Ablation of metals by ultrashort laser pulses. JOSA B. 1997;14(10):2716-2722.

[6] Mannion PT, Magee J, Coyne E, O’connor GM, Glynn TJ. The effect of damage accumulation behaviour on ablation thresholds and damage morphology in ultrafast laser micro-machining of common metals in air. Applied Surface Science. 2004;233(1-4):275-287.

[7] Abdelmalek A, Bedrane Z, Amara EH. Thermal and non-thermal explosion in metals ablation by femtosecond laser pulse: classical approach of the two temperature model. In: Journal of Physics: Conference Series. 2018;987(1): p.012012. IOP Publishing. 
[8] Abdelmalek A, Bedrane Z, Amara EH, Sotillo B, Bharadwaj V, Ramponi R, Eaton S. Ablation of Copper Metal Films by Femtosecond Laser Multipulse Irradiation. Applied Sciences. 2018;8(10):1826.

[9] Gruner A, Schille J, Loeschner U. Experimental study on micro hole drilling using ultrashort pulse laser radiation. Physics Procedia. 2016;83:157-166.

[10] Cheng C, Xu X. Mechanisms of decomposition of metal during femtosecond laser ablation. Physical Review B. 2005;72(16):165415.

[11] Yang J, Zhao Y, Zhu X. Theoretical studies of ultrafast ablation of metal targets dominated by phase explosion. Applied Physics A. 2007;89(2):571-578.

[12] Rethfeld B, Sokolowski-Tinten K, Von Der Linde D, Anisimov SI. Timescales in the response of materials to femtosecond laser excitation. Applied Physics A. 2004;79(4-6):767-769.

[13] Ren Y, Cheng CW, Chen JK, Zhang Y, Tzou DY. Thermal ablation of metal films by femtosecond laser bursts. International Journal of Thermal Sciences. 2013;70:32-40.

[14] Huang J, Zhang Y, Chen JK. Ultrafast solid-liquid-vapor phase change in a thin gold film irradiated by multiple femtosecond laser pulses. International Journal of Heat and Mass Transfer. 2009;52(13-14):30913100.

[15] Kerse C, Kalaycıoğlu H, Elahi P, Çetin B, Kesim DK, Akçaalan Ö, Yavaş S, Aşık MD, Öktem B, Hoogland H, Holzwarth R. Ablation-cooled material removal with ultrafast bursts of pulses. Nature. 2016;537(7618):84

[16] Von der Linde D, Sokolowski-Tinten K, Bialkowski J. Laser-solid interaction in the femtosecond time regime. Applied Surface Science. 1997;109:1-10.

[17] Chowdhury IH, Xu X. Heat transfer in femtosecond laser processing of metal. Numerical Heat Transfer: Part A: Applications. 2003;44(3):219-232.

[18] Cheng CW, Wang SY, Chang KP, Chen JK. Femtosecond laser ablation of copper at high laser fluence: Modeling and experimental comparison. Applied Surface Science. 2016;361:41-48.

[19] Kotsedi L, Kaviyarasu K, Fuku XG, Eaton SM, Amara EH, Bireche F, Ramponi R, Maaza M. Two temperature approach to femtosecond laser oxidation of molybdenum and morphological study. Applied Surface Science. 2017;421:213-219.

[20] Jia X, Zhao X. Numerical study of material decomposition in ultrafast laser interaction with metals. Applied Surface Science. 2019;463:781-790.

(C) 2019 by the author(s). This work is licensed under a Creative Commons Attribution 4.0 International License (http://creativecommons.org/licenses/by/4.0/). Authors retain copyright of their work, with first publication rights granted to Tech Reviews Ltd. 